\title{
Using Constraints to Building Version Spaces
}

\author{
Michèle Sebag
}

\author{
LMS-CNRS URA 317, Ecole Polytechnique, 91128 Palaiseau Cedex, France
}

\begin{abstract}
Our concern is building the set $\mathrm{G}$ of maximally general terms covering positive examples and rejecting negative examples in propositional logic.

Negative examples are represented as constraints on the search space. This representation allows for defining a partial order on the negative examples and on attributes too. It is shown that only minimal negative examples and minimal attributes are to be considered when building the set $\mathrm{G}$. These results hold in case of a non-convergent data set.

Constraints can be directly used for a polynomial characterization of $\mathrm{G}$.

They also allow for detecting erroneous examples in a data set.
\end{abstract}

\section{Introduction}

The Version Space frame defines two bounds in the search space in empirical inductive learning [7] : the upper bound, set $\mathrm{G}$, includes terms maximally general rejecting negative examples; the lower bound, set $S$, includes terms maximally specific covering positive examples. Many works in the machine learning field shows how inspiring this frame is : to mention but a few, Smith and Rosenbloom [11] show that in propositional logic, in the case of a convergent data set (leading to $S=G$ ), learning only needs to consider those negative examples that are near-misses, in the sense defined by Winston [12]. H. Hirsh [5] defines a set of operations on Version Spaces and studies their computational complexity within a propositional formalism.

This paper focuses on building set $\mathrm{G}$ from positive and negative examples in propositional logic. Our motivations for building set $\mathrm{G}$ are both cognitive and pragmatic. From a cognitive point of view, human learning seems to perform specialization only when forced to by negative examples or instructors [9]. The practical advantages of such doing are clear : when learning from a few examples ${ }^{1}$, specific learning leads to concepts of little further applicability.

But building set $\mathrm{G}$ is critical [2] : within a formalism as simple as the boolean one, its size may be exponential with respect to the number of attributes [4]. We propose, following the line of [5] and [8], a new formalization of the negative examples so to deal with the exponential size problem. Negative examples are formalized as constraints on the generalization ; a partial order on the negative examples is then derived. Therefore this formalisation results in extending the notion of near-miss and the polynomial results of [11] to a non convergent data

\footnotetext{
1 This is the case for children ; this is the case also for many industrial problems where gathering examples is expensive.
} 
set. It also allows for pruning the attributes of the problem domain. Moreover, it allows detection of erroneous examples in a ML-Smart -like way [1].

This paper is organized as follows. Section 2 formalizes negative examples as constraints on generalization. A partial order on negative examples is derived from the constraints ; it is shown that only minimal negative examples are necessary to learning. Section 3 defines the partial order on attributes derived from the constraints and shows that only minimal attributes are to be explored when building G. Section 4 focuses on expressing $G$ and classifying a further case. The complexity of the proposed classification is in $\mathcal{O}(K \times P \times N)$, where $\mathrm{K}$ is the number of attributes, $\mathrm{P}$ the number of positive examples and $\mathrm{N}$ the number of negative examples. Section 5 describes how to detect erroneous examples by using constraints. Last, section 6 briefly reviews some related works.

\section{From negative examples to constraints}

The problem domain is described by $K$ attributes $x_{1}, . ., x_{K} ;$ attributes are linear i.e. integer- or real-valued [6], or valued in a tree-structured domain. Given a conjunctive term $\mathrm{S}$ and a set of negative examples $C e_{1}, \ldots C e_{N}$, the solution space is that of conjunctive terms covering $S$ and rejecting any negative example. In this section, our goal is to propose a representation of the negative examples enabling ordering and pruning of both the negative examples (2.4) and the attributes (in section 3 ).

\subsection{A negative example induces a constraint}

Let us consider a toy problem for the purpose of illustration. The concept to learn is that of Hero; attributes are the name of the person, its favorite color and the number of questions $\mathrm{s} /$ he asks :

\begin{tabular}{|c|c|c|c|}
\hline & Name & Color & Nb Questions \\
\hline$\overline{E x}$ & Arthur & Blue & 3 \\
\hline$C e_{1}$ & Ganelon & Grey & 7 \\
\hline$C e_{2}$ & Iago & Yellow & 4 \\
\hline$C e_{3}$ & Bryan & Green & 10 \\
\hline$C e_{4}$ & Triboulet & Cream & 5 \\
\hline
\end{tabular}

Attribute $\mathrm{Nb}$-Questions is linear; Name and Favorite Color are tree-structured :

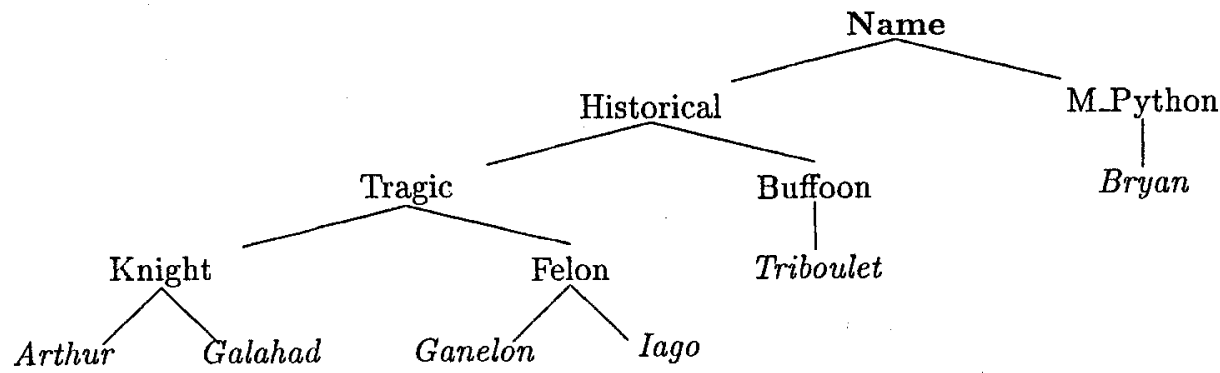




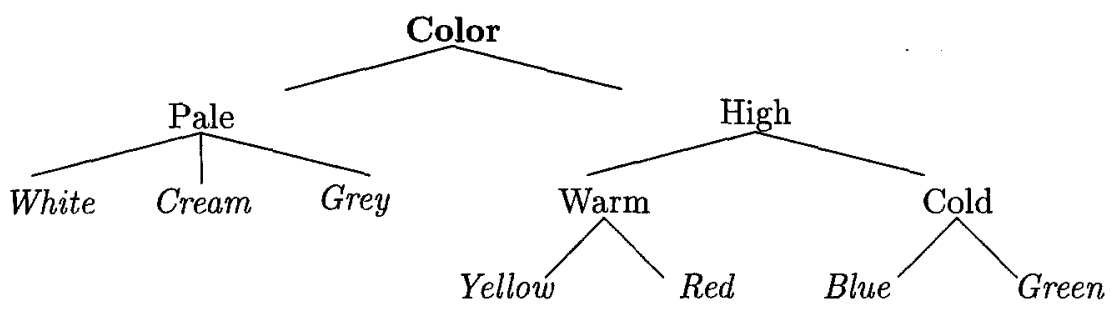

Any discriminant generalization of example $E x$ must reject negative example $C e_{1}$. In the case of attribute Color, the most general value covering Blue and rejecting Grey is High. The corresponding selector [6] rejecting $C_{1} e_{1}$ thus is : $[$ Color $=H i g h]$. The disjunction of the most general selectors covering $E x$ and rejecting $C e_{1}$ is :

$$
[\text { Name }=\text { Knight }] \bigvee[\text { Color }=\text { High }] \bigvee\left[N b_{-} \text {Questions }=[0,6]\right]
$$

This disjunction can be thought of as a constraint upon the generalization of $E x$ : any term in the solution space must satisfy this logical constraint.

More generally, such a constraint upon the generalization of any positive term can be derived from any negative example. After proposing a representation of such constraints, we focus on their pruning (2.4) and handling in order to express G (4.1) or classify a further case (4.2).

\subsection{Formalizing Constraints}

In the following, the notation $x(T)$ stands for the value of attribute $x$ in term $T ; x(T)$ thus is a qualitative value in the case of a tree-structured attribute, and an interval or a single value in the case of a linear attribute. The $G$ set associated to a positive term $S$ and negative examples $C e_{1}, \ldots, C e_{N}$ is noted $\mathrm{G}\left(S, C e_{1}, . . C e_{N}\right)$.

Now, for any negative example $C e_{j}$, for any attribute $x_{i}$, - Let $E_{i, j}$ be, if it exists, the most general value (respectively the widest interval) such that it covers (resp. includes) value $x_{i}(S)$ and does not cover (resp. include) value $x_{i}\left(C e_{j}\right)$ if attribute $x_{i}$ is qualitative (resp. linear).

- Let $I(j)$ be the set of attributes such that value $E_{i, j}$ is defined. $I(j)$ denotes the set of possibly guilty attributes, according to the terminology of [11].

By definition, the most general selectors covering $\mathrm{S}$ and rejecting $C e_{j}$ (if we restrict to operator ' $=')$, are the $\left[x_{i}=E_{i, j}\right]$, for $x_{i}$ in $I(j)$. So, one has :

$$
G\left(S, C e_{j}\right)=\bigvee_{x_{i} \in I(j)}\left[x_{i}=E_{i, j}\right]
$$

The above expression, called constraint induced by $C e_{j}$ on the generalization of $S$, is given an extensional representation. 
Let $O_{i}$ be the domain of attribute $x_{i}$, and let $\Omega$ denote the cross product of domains $O_{i}: \Omega=O_{1} \times \ldots \times O_{K}$. A constraint may then be represented as a subset of $\Omega$.

Definition (constraint) :

Given term $S$, one associates to any negative example $C e_{j}$ the subset of $\Omega$ denoted Constraint $\left(S, C e_{j}\right)$, defined by :

$$
\begin{gathered}
\text { Constraint }\left(S, C e_{j}\right)=\left(V_{i, j}\right)_{i=1}^{K} \\
\text { where } V_{i, j}=\left\{\begin{array}{ll}
E_{i, j} \text { if } E_{i, j} \text { is defined } \\
\phi & \text { otherwise }
\end{array} \quad\left(\Leftrightarrow x_{i} \in I(j)\right)\right.
\end{gathered}
$$

The empty value $\phi$ is assumed more specific than any value $V_{i}$ in any domain $O_{i}$.

The negative examples described in 2.1 give rise to the following constraints :

\begin{tabular}{l|ccc} 
& Name & Color & Nb Questions \\
\hline Constraint $\left(E x, C e_{1}\right)$ & Knight_Name & High_Color & {$[0,6]$} \\
Constraint $\left(E x, C e_{2}\right)$ & Knight_Name & Cold_Color & {$[0,3]$} \\
Constraint $\left(E x, C e_{3}\right)$ & Historical_Name & Blue & {$[0,9]$} \\
Constraint $\left(E x, C e_{4}\right)$ & Tragic & High_Color & {$[0,4]$}
\end{tabular}

\subsection{Ordering Constraints and Negative Examples}

The partial order relations defined on domains $O_{i}$ classically induce a partial order relation on their cross-product $\Omega$, denoted $\leq_{\Omega}$. One has :

$$
\left(\left(V_{i}\right)_{i=1}^{K} \leq_{\Omega}\left(W_{i}\right)_{i=1}^{K}\right) \Longleftrightarrow\left(\forall i=1 . . K, V_{i} \leq W_{i}\right)
$$

where $V_{i} \leq W_{i}$ means that value $V_{i}$ is covered by value $W_{i}$, if attribute $x_{i}$ is tree-structured, and that interval $V_{i}$ is included in interval $W_{i}$ if $x_{i}$ is linear.

This order relation enables comparing constraints and thus, negative examples.

Definition (nearest-miss):

Given term $S$ and negative examples $C e_{1}, \ldots C e_{N}, C e_{i}$ is called nearest miss to $S$ iff Constraint $\left(S, C e_{i}\right)$ is minimal with respect to the order relation $\leq_{\Omega}$ among Constraints $\left(S, C e_{j}\right), j=1 . . N$.

Negative example $C e_{2}$ is a nearest miss; $C e_{4}$ is not for we have :

$$
\text { Constraint }\left(E x, C e_{2}\right) \leq{ }_{\Omega} \text { Constraint }\left(E x, C e_{4}\right)
$$

(Knight is less general than Tragic; Cold is less general than High; last, one has $[0,3] \subset[0,4])$. This shows that a nearest-miss is not necessarily a near-miss: $\mathrm{Ce}_{2}$ is discriminated from $\mathrm{Ex}$ by more than one attribute. 


\subsection{Pruning negative examples}

Given the above definition, a result parallel to that of [11] holds : bottom-up learning only needs positive examples and nearest-miss negative examples.

Proposition 1:

Given positive term $\mathrm{S}$ and negative examples $C e_{1}, \ldots C e_{N}$, assume without loss of generality that nearest-misses are examples $C e_{1}, \ldots C e_{L}, L \leq N$. Then

$$
G\left(S, C e_{1}, \ldots C e_{N}\right)=G\left(S, C e_{1}, \ldots C e_{L}\right)
$$

Proof.

- We first associate to any $\omega$ in $\Omega, \omega=\left(V_{i}\right)_{i=1}^{K}$, the disjunction of selectors $\left[x_{i}=V_{i}\right]$, for $V_{i}$ not empty :

$$
\omega \in \Omega \rightarrow g(\omega)=\bigvee_{i / V_{i} \neq \phi}\left[x_{i}=V_{i}\right]
$$

It is straightforward to show that (where $\leq$ stands for 'less general than') :

$$
\forall \omega_{1}, \omega_{2} \in \Omega,\left(\omega_{1} \leq_{\Omega} \omega_{2}\right) \Longleftrightarrow\left(g\left(\omega_{1}\right) \leq g\left(\omega_{2}\right)\right)
$$

- By definition, one has

$$
G\left(S, C e_{i}\right)=g\left(\text { Constraint }\left(S, C e_{i}\right)\right)
$$

and

$$
G\left(S, C e_{1}, \ldots, C e_{N}\right)=\bigwedge_{i=1}^{N}\left(g\left(\text { Constraint }\left(S, C e_{i}\right)\right)\right)
$$

- For any negative example $C e_{j}$ which is not a nearest-miss, $(j \geq L)$, there is a nearest-miss $C e_{i}$ with $\operatorname{Constraint}\left(S, C e_{i}\right) \leq_{\Omega} \operatorname{Constraint}\left(S, C e_{j}\right)$. Hence from (1), one has $g\left(\right.$ Constraint $\left.\left(S, C e_{i}\right)\right) \leq g\left(\right.$ Constraint $\left.\left(S, C e_{j}\right)\right)$. So

$$
g\left(\text { Constraint }\left(S, C e_{i}\right)\right) \bigwedge g\left(\text { Constraint }\left(S, C e_{j}\right)\right)=g\left(\text { Constraint }\left(S, C e_{i}\right)\right)
$$

And

$$
G\left(S, C e_{1}, . . C e_{L}\right)=G\left(S, C e_{1}, . . C e_{L}\right) \bigwedge G\left(S, C e_{L+1}, . . C e_{N}\right)=G\left(S, C e_{1}, . . C e_{N}\right)
$$

This proposition then extends the result of [11] in case of a non convergent data set ${ }^{2}$. Therefore, negative examples that are not near-misses can be pruned without any loss of information.

\footnotetext{
${ }^{2}$ From [11], we have then : a data set is convergent iff there are at most $N$ minimal constraints, each of them involving a single attribute.
} 


\subsection{Complexity}

The notations used are that of Smith and Rosenbloom [11] : $K$ denotes the number of attributes, $P$ the number of positive examples and $N$ the number of negative examples. It is assumed that exploring the domain hierarchy of any attribute can be done in constant time ; then

- Following [11], the complexity of building set $\mathrm{S}$ is in $\mathcal{O}(P \times K)$. Storing $\mathrm{S}$ requires a $\mathcal{O}(K)$ memory size.

- The constraints building is in $\mathcal{O}(N \times K)$ according to 2.2 ; their update when $\mathrm{S}$ is generalized is in $\mathcal{O}(N \times K)$ too $^{3}$. The incremental building of the constraints so is in $\mathcal{O}(P \times N \times K)$. Their storage is in $\mathcal{O}(N \times K)$.

- The constraints pruning, according to definition 2.3, is in $\mathcal{O}\left(N^{2} \times K\right)$.

\section{Ordering and Pruning Attributes}

A partial order on the attributes $x_{1}, \ldots x_{K}$ of the problem domain can be derived from a set of constraints. It is shown that only minimal attributes with respect to this order are to be explored when building the $\mathrm{G}$ set.

\subsection{Definition}

Given constraints $C_{1}, \ldots, C_{L}$, for any attribute $x_{i}$, we denote $O_{i}^{*}$ the set of values $x_{i}\left(C_{m}\right)$ for $m=1$..L. This set $O_{i}^{*}$ induces a partition over the set of constraints, denoted $\mathcal{P}_{i}$ : two constraints belong to the same subset if they have same value for attribute $x_{i}$. Subset $\mathcal{E}_{i, k}$ in partition $\mathcal{P}_{i}$ is the set of constraints $C_{m}$ such that $x_{i}\left(C_{m}\right)$ equals value $U_{i, k}$, belonging to $O_{i}^{*}$.

So, if we consider the set of minimal constraints $C_{1}, \ldots C_{3}$ :

\begin{tabular}{c|ccc} 
& Name & Color Nb Questions \\
\hline$C_{1}$ & Knight & High & {$[0,6]$} \\
$C_{2}$ & Knight & Cold & {$[0,3]$} \\
$C_{3}$ & Historical & Blue & {$[0,9]$}
\end{tabular}

we have $O_{1}^{*}=\{$ Knigt, Historical $\}$, which induces the partition $\mathcal{P}_{1}=\left\{\left\{C_{1}, C_{2}\right\},\left\{C_{3}\right\}\right\}$

The partitions over a given domain are partially ordered ; their order relation noted $\leq$, classically is defined by :

$$
\left(\mathcal{P}_{1} \leq \mathcal{P}_{2}\right) \Leftrightarrow\left(\forall \mathcal{E}_{1, i} \in \mathcal{P}_{1}, \exists \mathcal{E}_{2, j} \in \mathcal{P}_{2} / \mathcal{E}_{1, i} \subset \mathcal{E}_{2, j}\right)
$$

If two partitions $\mathcal{P}_{i}$ and $\mathcal{P}_{j}$ are such that $\mathcal{P}_{i} \leq \mathcal{P}_{j}$, we can define an application $\varphi_{i, j}$ from $O_{i}^{*}$ to $O_{j}^{*}$ which associates to value $U_{i, k}$ value $U_{j, l}$ such that for

\footnotetext{
${ }^{3}$ For every attribute $x_{i}$, for every constraint $C_{j}$, if $x_{i}$ is in $I(j)$ (value $E_{i, j}$ is defined), it is checked whether $E_{i, j}$ still is more general than $x_{i}(S)$. If not, $x_{i}$ is removed from set $I(j)$. If set $I(j)$ is empty for a constraint $C_{j}$, negative example $C e_{j}$ is no longer discriminated from $S$, and the version space fails.
} 
any constraint $C_{m}, x_{i}\left(C_{m}\right)=U_{i, k} \Rightarrow x_{j}\left(C_{m}\right)=U_{j, l}$.

For instance, the partition $\mathcal{P}_{3}=\left\{\left\{C_{1}\right\},\left\{C_{2}\right\},\left\{C_{3}\right\}\right\}$ derived from attribute $N b_{-}$Questions is finer than the partition $\mathcal{P}_{1}$. The corresponding application $\varphi_{3,1}$ is defined by :

$$
\begin{gathered}
\varphi_{3,1}([0,6])=\varphi_{3,1}([0,3])=\text { Knight } \\
\varphi_{3,1}([0,9])=\text { Historical }
\end{gathered}
$$

This partial order allows for defining a partial order on attributes :

Definition (finer attribute):

Given a set of constraints $C_{1}, \ldots, C_{L}$, attribute $x_{i}$ is finer than attribute $x_{j}$, noted $x_{i} \leq_{\text {att }} x_{j}$ iff :

- Sets $O_{i}^{*}$ and $O_{j}^{*}$ are totally ordered with respect to set inclusion ${ }^{4}$.

- The partition induced by $x_{i}$ is finer than the partition induced by $x_{j}: \mathcal{P}_{i} \leq \mathcal{P}_{j}$

- Function $\varphi_{i, j}$ defined above is monotonic from $O_{i}^{*}$ into $O_{j}^{*}$

$$
\forall U_{1}, U_{2} \in O_{i}^{*},\left(\left(U_{1}<U_{2}\right) \Rightarrow\left(\varphi_{i, j}\left(U_{1}\right) \leq \varphi_{i, j}\left(U_{2}\right)\right)\right)
$$

- For every $k$, constraint $C_{k}$ involves attribute $x_{i}\left(x_{i}\left(C_{k}\right) \neq \phi\right)$ iff it also involves attribute $x_{j}$.

Attribute $\mathrm{Nb}_{-}$Questions is finer than attribute Name: the set of values $O_{3}^{*}=\{[0,3],[0,6],[0,9]\}$ is ordered and $\varphi_{3,1}$ is monotonic.

\subsection{Pruning attributes}

The partial order defined on the attributes enables a result parallel to that of the section 2.4 : only minimal attributes with respect to this order are to be explored when building G.

Proposition 2:

Let $x_{i}$ and $x_{j}$ be two attributes such that $x_{i} \leq_{a t t} x_{j}$.

Let $\mathrm{G}\left(S, C_{1}, \ldots C_{L}\right)$ denote the set of maximally general terms covering term $\mathrm{S}$ and satisfying constraints $C_{1}, \ldots C_{L}$. Let G' be the subset of $\mathrm{G}$ given by the terms not involving attribute $x_{j}$.

For any term $T$ in $\mathrm{G}^{\prime}$, one defines term $T^{*}$ by :

- if $T$ does not involve attribute $x_{i}, T^{*}=T$.

- Otherwise, let $\left[x_{i}=V_{i}\right]$ be the selector involving $x_{i}$ in $T^{5}$.

- If $V_{i}$ is such that $V_{i}=\sup \left\{V_{k} / \phi_{i, j}\left(V_{k}\right)=\phi_{i, j}\left(V_{i}\right)\right\}$, then $T^{*}$ is the term obtained by replacing selector $\left[x_{i}=V_{i}\right]$ in $T$ by selector $\left[x_{j}=\right.$ $\left.\varphi_{i, j}\left(V_{i}\right)\right]$.

- Otherwise $T^{*}=T$.

\footnotetext{
${ }^{4}$ If $x_{i}$ is a tree-structured attribute then $O_{i}^{*}$ is totally ordered : all values in $O_{i}^{*}$ can be compared for they all are more general than $x_{i}(S)$ by construction. If $x_{j}$ is a linear attribute, $O_{j}^{*}$ is not necessarily totally ordered w.r.t. set inclusion.

${ }^{5}$ This selector is unique : only the most specific value $V_{i}$ is retained.
} 
Then any term in $\mathrm{G}$ either belongs to $\mathrm{G}^{\prime}$, or is a $T^{*}$ for some $\mathrm{T}$ in $\mathrm{G}^{\prime}$.

The proof is given in appendix.

In practice, set $G^{\prime}$ is obtained by considering minimal attributes only. (The attributes pruning thus only takes place during the expensive phase of the $G$ building.) Then, $\mathrm{G}$ is obtained from $\mathrm{G}^{\prime}$ by making straightforward use of functions $\varphi_{i, j}$. In the example, only attributes $C_{\text {olor }}$ and $N b_{-} Q$ Questions are considered to build $\mathrm{G}^{\prime}$; the terms in set $\mathrm{G}^{\prime}$ are :

$T_{1}=[$ Color $=$ Blue $]$

$T_{2}=[$ Color $=$ Cold $] \wedge\left[N b_{-}\right.$Questions $\left.=[0,9]\right]$

$T_{3}=[$ Color $=$ High $] \wedge\left[N_{-} b_{\text {Questions }}=[0,3]\right]$

Terms $T_{2}$ and $T_{3}$ give rise to terms $T_{2}^{*}$ and $T_{3}^{*}$

$T_{2}^{*}=[$ Color $=$ Cold $] \wedge[$ Name $=$ Historical_Name $]$

$T_{3}^{*}=[$ Color $=$ High $] \wedge[$ Name $=$ Knight_Name $]$

\subsection{Complexity}

Pruning attributes involves, for any (ordered) pair of attribute $\left(x_{i}, x_{j}\right)$ :

- Building application $\varphi_{i, j}$ from $O_{i}^{*}$ into $O_{j}^{*}$; the size of $O_{i}^{*}$ is upper bounded by the number of constraints, (which is upper bounded by the number of negative examples $N$ ) and by the number of values in $O_{i}$. Hence, if $L$ denotes the maximum number of values in any $O_{i}$, this step is in $\mathcal{O}(\min (N, L))$. The storage of $\varphi_{i, j}$ also is in $\mathcal{O}(\min (N, L))$.

- Checking the monotonicity of $\varphi_{i, j}$, which is in $\mathcal{O}(\min (N, L) \log (\min (N, L)))$.

Finally, the complexity of the attributes pruning is in $\mathcal{O}\left(K^{2} \times \min (N, L) \log (\min (N, L))\right)$.

\section{Building G or Classifying ?}

This section addresses the characterization of $\mathrm{G}$ from the constraints.

\subsection{Building G}

In a first step, $\mathrm{S}$ is built from the set of positive examples ; constraints are built and updated as detailed in 2.2 and 2.5 .

Constraints are then explored in order to build the terms in G. Here is the pseudo-code of the constrained generalization algorithm ${ }^{6}$. Array Selector $[i]$ stores the active selector of constraint $C_{i} ; i_{0}$ is the index of the current constraint.

Initialize()

For $\mathrm{i}=1 . \mathrm{N}$

${ }^{6}$ This algorithm is implemented in $\mathrm{C}^{++}$. 


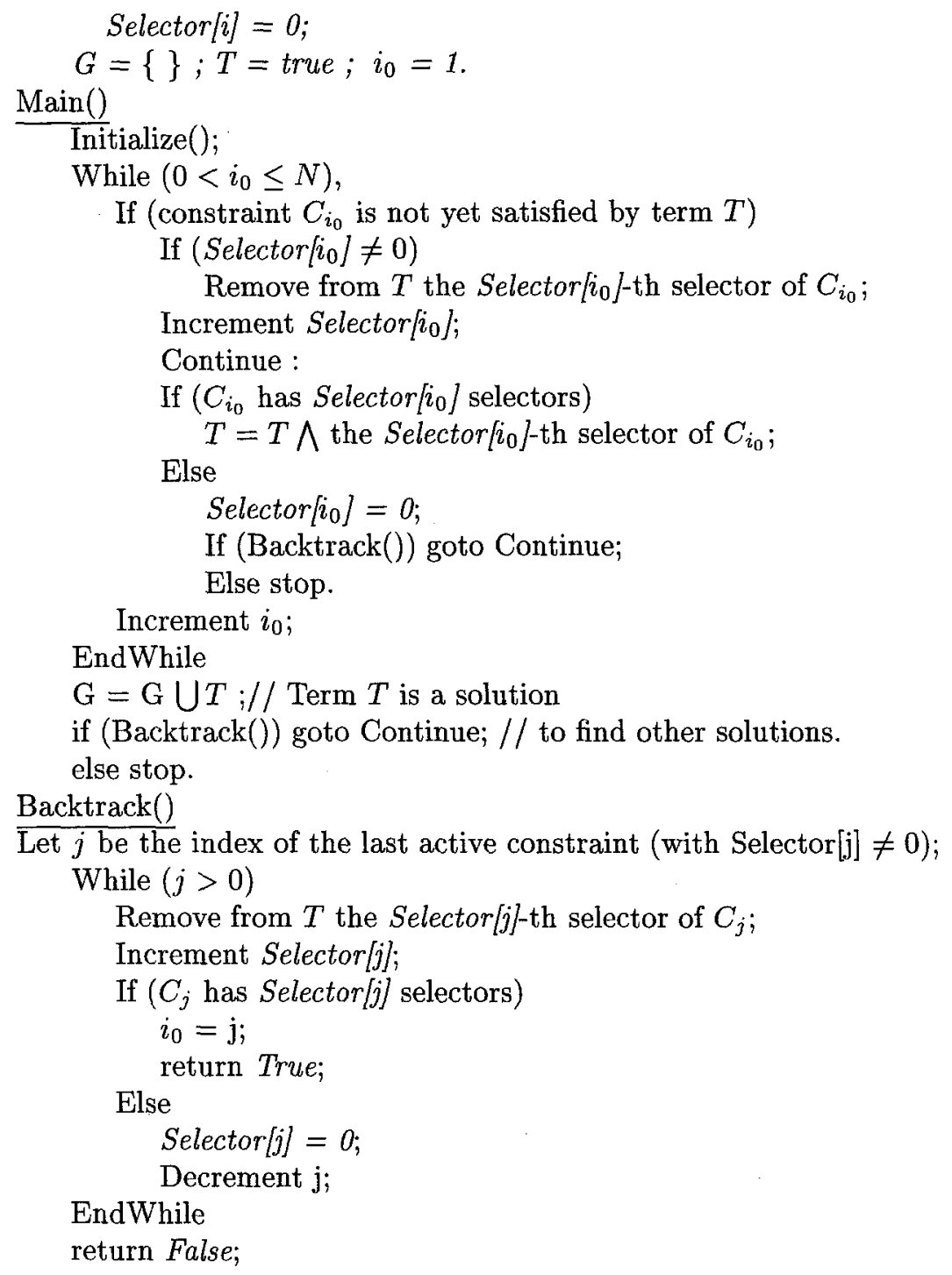

This procedure allows to find all terms in $\mathrm{G}$; however, terms found may be not all maximally general : a selector added to satisfy a given constraint may become useless because of a selector added further on and satisfying also this constraint.

So one has to check whether a term $T$ is maximally general or not. This is done by building $G\left(T, C e_{1}, . . C e_{N}\right)$; T is maximally general iff $G\left(T, C e_{1}, . . C e_{N}\right)=$ $T$.

In spite of the pruning of the search space enabled by constraints, the number of conjunctive terms in G may still be exponential with respect to the number 
of attributes. However, the constraints are sufficient to characterize $G$ with a polynomial complexity.

\subsection{Using constraints to classify}

Let $\mathrm{E}$ be the description of a further case. The diagnosis function is as usual given by

$$
\text { Diagnosis }(E)= \begin{cases}\text { True } & \text { if } E \leq S \\ \text { False } & \text { if } E \leq G \\ \text { Unknown otherwise }\end{cases}
$$

The only point is checking whether $\mathrm{E}$ belongs to $\mathrm{G}$, i.e. whether $\mathrm{E}$ satisfies all constraints Constraint $\left(S, C e_{i}\right)$. This can be done with a complexity $\mathcal{O}(K \times N)$ (still assuming that exploring the generalization hierarchy of any attribute is done in constant time):

Therefore, the proposed formalization provides the user with a polynomial characterization of $\mathrm{G}$, even in case of a non-convergent data set. This result is discussed with reference to that of $\mathrm{H}$. Hirsh [5] in the last section.

\section{Detecting erroneous examples}

Dealing with noisy data has long been recognized an unavoidable task in machine learning [3]. However, detecting and rejecting outliers and/or erroneous examples could ease greatly the learning task [10]. This section deals with detecting erroneous examples in the data set. More precisely, it gives sufficient conditions for an example to be erroneous. An example is said to be erroneous if either its description or its conclusion differ from what it should be.

Negative examples are represented as constraints with respect to a positive term to generalize; this representation holds, be this positive term either the actual $\mathrm{S}$, or any positive example $E x_{i}$ in the data set. Let $C\left(E_{i}, C e_{k}\right)$ denote the constraint put by negative example $C e_{k}$ upon the generalization of positive example $E_{i}$. This constraint is a boolean function computable on the problem domain. Should positive example $E_{j}$ satisfy constraint $C\left(E_{i}, C e_{k}\right)$ ? Yes : if $E_{j}$ belongs to concept $\mathrm{C}$, then $E_{j}$ belongs to set $\mathrm{G}$, and then it belongs to any set $\mathrm{G}\left(E_{i}, C e_{1}, \ldots, C e_{N}\right)$ in case of a conjunctive concept $\mathrm{C}$. So, let us denote $\delta(i, j, k)$ the boolean value $C\left(E_{i}, C e_{k}\right)\left(E_{j}\right)$; this boolean should be true for any positive example $E_{j}$.

Assume now that $\delta(i, j, k)$ is false : then, according to the above discussion, either $E_{i}$ or $E_{j}$ or $C e_{k}$ is erroneous, i.e. has a corrupted description or conclusion. This fact only gives a hint ; but one can appreciate where the problem eventually comes from, by aggregating hints and considering all $\delta(i, j, k)$.

The procedure is inspired from the one exposed in [1]. Bergadano et al. state that along the specialization process, the number of examples belonging to the extension of the current term is to decrease ; the point is that, when specialization is based on a litteral defined by the domain theory - and if this definition is too specific, then this decreasing is much higher than the average decreasing of 
the extension size, averaged along the specialization process. When an unusual decrease is observed, the current predicate and its definition are submitted for correction to the expert. In the same line, we associate to example $E x_{i}$ the number of booleans $\delta(i, j, k)$ that are false, for $j$ ranking from 1 to $\mathrm{P}$ and $k$ from 1 to N. Let $\sigma\left(E x_{i}\right)$ denote this sum. This allows to order positive examples by ascending order of "suspicion" : the greater $\sigma\left(E x_{i}\right)$, the more likely $E x_{i}$ is erroneous. Similarly, one can associate to a negative example $C e_{k}$ the number of $\delta(i, j, k)$ that are false, for $i$ and $j$ ranking from 1 to $\mathrm{P}$. This quantity allows for ordering negative examples by ascending order of suspicion.

Of course, the final decision to reject an example as erroneous belongs to the expert ; the only crisp information provided by our approach is to state that, given a boolean $\delta(i, j, k)$ false, one at least among examples $E x_{i}, E x_{j}$ and $C e_{k}$, is erroneous. In practice, we proceed as follows : the expert provides the system with a rate of erroneous examples. Then, until this rate of examples has been rejected, or until the stack is empty, the positive and negative examples maximizing function $\sigma$ are proposed to the expert. If they are discarded by the expert, function $\sigma$ is updated; otherwise, the next positive and negative examples maximizing $\sigma$ are considered.

\section{Related works}

\subsection{An exponential size}

Among the related works, we must first mention Haussler [4] who showed that the number of conjunctive terms in set $G$ could be exponential with respect to the number of attributes. The example is as follows :

The problem domain is $\{0,1\}^{2 m}$. One is given positive example $E x$, whose all components are true, and $m$ negative examples $C e_{i}, i=1 . . m$; components of $C e_{i}$ are all true, except the $i$-th and the $(m+i)$-th.

Any negative example $C e_{i}$ leads to specialise set $G$; this specialization may be done along any feature discriminating $C e_{i}$ from $E x=S$, i.e. one of attributes $i$ or $m+i$. The number of choices (and of conjunctive terms in G) is multiplied by 2 at each negative example. The final number of elements in $G$ is thus $2^{m}$.

Many strategies have been proposed so to deal with that number of choices and terms.

\subsection{Using near-misses}

Smith and Rosenbloom [11] first consider the negative examples which are discriminated from $\mathrm{S}$ by only one attribute (so there is no choice for specialization). Such negative examples are called near-misses, according to Winston [12]. A major result of [11] is to show that when the data set is convergent, learning only needs positive examples and near-miss negative examples to converge. Accordingly, they propose an algorithm linear with respect to the number of attributes $K$, the number of positive examples $P$ and the number of negative examples $N$ : 
Negative examples are stocked in a waiting list.

When set $\mathrm{S}$ is generalized from a positive example, the waiting list is scanned : if there is a negative example which is a near-miss (i.e. with exactly one attribute discriminating this example from $S$ ), then $G$ is specialized with respect to this guilty attribute so to reject the negative example.

This way, the memory size required is in $\mathcal{O}((N+2) \times K)$ (stocking of $\mathrm{S}$, $\mathrm{G}$ and the waiting list).

This process leads to $\mathrm{G}=\mathrm{S}$ if the data set is convergent. Otherwise, after, having considered all positive examples and near-miss negative examples, the usual Candidate Elimination Algorithm [7] is used to update set G from the remaining negative examples.

\subsection{Another representation of the G set}

H. Hirsh [5] proposes to represent a Version Space by $[S, \mathcal{N}]$ where $\mathcal{N}$ stands for the list of negative examples. For conjunctive tree-structured languages, this representation supports a polynomial computation of some functions defined on a Version Space:

- Collapse : when data are inconsistent, or the description langage does not allow to describe the concept to learn ; Collapse is true if $\mathrm{S}$ is empty ; (see Update below);

- Converge : in case of convergence, from [11] there is a near-miss for any attribute in list $\mathcal{N}$;

- Update given new example $E$ : if $E$ is negative it is added to list $\mathcal{N}$ and elements in $\mathrm{S}$ covering it are removed ; otherwise $E$ is used to generalize $\mathrm{S}$, and resulting terms covering some example in $\mathcal{N}$ are removed ;

- Classify a new case $E$ : if $E$ satisfies any term in $S$, then it belongs to the concept ; otherwise, compute the Version Space that would result if $E$ were a positive example ; if this new Version Space collapses, $E$ does not actually belong to the concept; otherwise, the diagnosis is unknown.

The drawback of this representation is that maintaining the list of negative examples gives few hints to what G could be. Addressing this remark, J. Nicolas [8] proposes a disjunctive formalization such that the $\mathrm{G}$ set induced by a single negative example is represented by a single term. The trouble comes from intersecting several Version Spaces when several negative examples are considered. This operation is very expensive; the actual number of (disjunctive) terms may still be exponential.

\subsection{Discussion}

Our approach is very near from that of $\mathrm{H}$. Hirsh, with a slightly higher complexity of our update (learning) phase ; the complexity of the classifying phase is equivalent to that of Hirsh : it is equivalent to check whether case $E$ satisfies 
Constraint $\left(S, C e_{i}\right)$, or whether the generalization of $E$ and $S$ covers negative example $C e_{i}$.

From the intelligibility standpoint the constraint derived from a negative example is more general and thus understandable by the expert than the negative example itself (furthermore, by pruning the constraints the number of useful informations can be much decreased). So, the presented approach achieves some trade-off between efficiency and understandability. But the major advantage of our formalization compared to that of $\mathrm{H}$. Hirsh, it that it enables to detect the erroneous examples by an all-at-once handling of the data.

Compared to the approach of J. Nicolas [8], the final expensive phase of the $G$ building is performed in a reduced search space : constraints allow for pruning both negative examples and attributes. This phase can also be completely escaped as shown in 4.2 .

\section{Summary and Perspectives}

Representing the negative examples as constraints on the generalization of a positive term enables to prune the negative examples and the attributes to be explored when building the $\mathrm{G}$ set.

This representation also allows for a computable polynomial characterization of $\mathrm{G}$; whatever the actual number of conjunctive terms in $\mathrm{G}$, this characterization is linear with respect to the number of attributes, the number of positive examples and the number of negative examples. The price to pay lies in the fact that a set of constraints is less understandable by the expert than a set of conjunctive terms. Last, our approach enables detecting erroneous examples.

Further research aims at extending this approach to learning a disjunctive concept. The constraints building applies, be the positive term considered the $\mathrm{S}$ set or any positive example $E x_{i}$. One may then consider the $\mathrm{G}$ set derived from a positive example $E x_{i}$ and the negative examples $C e_{1}, \ldots C e_{N}$, (the star of $E x_{i}$ by analogy with the star algorithm [6]). A next step is to cluster these stars, so to identify the (conjunctive) subconcepts involved in a disjunctive concept.

\section{References}

1. F. Bergadano, A Giordana, A Knowledge Intensive Approach to Concept Induction, ICML 1988, pp 305-317.

2. A. Bundy, B. Silver, D. Plummer, An analytical Comparizon of Some Rule Learning Programs, Artificial Intelligence, 27, 1985, pp 137-181.

3. P. Clark T. Niblett Induction in noisy domains Progress in machine learning, Proc. EWSL 1987, I. Bratko N. Lavrac Eds, Sigma Press.

4. D. Haussler, Quantifying Inductive Bias : AI Learnign Algorithms and Valiant's Learning Framework, Artificial Intelligence, 36, 1988, pp 177-221.

5. H. Hirsh, Polynomial-Time Learning with Version Spaces, Proc. National Conference on Artificial Intelligence, 1992 pp 117-122. 
6. Michalski R.S. A theory and methodology for inductive learning Machine Learning: An Artificial Intelligence Approach, I, R.S. Michalski, J.G. Carbonnell, T.M. Mitchell Eds, Springer Verlag, (1983), p 83-134.

7. T.M. Mitchell, Generalization as Search, Artificial Intelligence Vol 18, pp 203-226, 1982.

8. J. Nicolas, Une Représentation Efficace pour les Espaces de Version, JFA 1993.

9. J. Piaget, Six études de psychologie, Denoel 1964.

10. R. Quinlan, The effect of noise on concept learning Machine Learning: An Artificial Intelligence Approach, I, R.S. Michalski, J.G. Carbonnell, T.M. Mitchell Eds, Vol 2, Morgan Kaufman, 1986.

11. B. Smith, P. Rosenbloom, Incremental non-backtracking focussing : A polynomially- bounded generalization algorithm for version space, Proc. National Conference on Artificial Intelligence, 1990, pp 848-853.

12. P.H. Winston, Learning Structural Descriptions from Examples The Psychology of Computer Vision, P.H. Winston Ed, Mc Graw Hill, New York, 1975, pp 157-209.

\section{Appendix}

\section{Proposition 2 :}

$\overline{\text { Let } x_{i} \text { and } x_{j}}$ be two attributes such that $x_{i} \leq_{a t t} x_{j}$.

Let $\mathrm{G}\left(S, C_{1}, \ldots C_{L}\right)$ denote the set of terms maximally general covering term $\mathrm{S}$ and satisfying constraints $C_{1}, \ldots C_{L}$. Let $\mathrm{G}^{\prime}$ be the subset of $\mathrm{G}$ given by the terms not involving attribute $x_{j}$.

For any term $T$ in $\mathrm{G}^{\prime}$, one defines term $T^{*}$ by :

- if $T$ does not involve attribute $x_{i}, T^{*}=T$.

- Otherwise, let $\left[x_{i}=V_{i}\right]$ be the selector involving $x_{i}$ in $T$.

- If $V_{i}$ is such that $V_{i}=\sup \left\{V_{k} / \phi_{i, j}\left(V_{k}\right)=\phi_{i, j}\left(V_{i}\right)\right\}$, then $T^{*}$ is the term obtained by replacing selector $\left[x_{i}=V_{i}\right]$ in $T$ by selector $\left[x_{j}=\phi_{i, j}\left(V_{i}\right)\right]$.

- Otherwise $T^{*}=T$.

Then any term in $\mathrm{G}$ either belongs to $\mathrm{G}^{\prime}$, or is a $T^{*}$ for some $T$ in $\mathrm{G}^{\prime}$.

Proof.

A preliminary remark is the following : as set $O_{l}^{*}$ is totally ordered for $l=i$ or $j^{7}$, it induces a total order on partition $\mathcal{P}_{l}$ too. So selector $\left[x_{l}=U_{l, m}\right]$ enables to satisfy any constraint $C_{k}$ such that $U_{l, m} \leq x_{l}\left(C_{k}\right)$, and more generally, any constraint belonging to some $\mathcal{E}_{l, j}$ in $\mathcal{P}_{l}$, with index $\bar{j}$ greater than $m$.

$$
\text { Let } G^{\prime \prime}=\bigvee_{T \in G^{\prime}}\left(T \bigvee T^{*}\right)
$$

\section{A.1 $G \subset G^{\prime \prime}$}

Let $Z$ be a maximal term in $\mathrm{G}$.

- We first show that $Z$ involves at most one among attributes $x_{i}$ or $x_{j}$. Let us suppose that $Z$ includes two selectors $\left[x_{i}=V_{i}\right]$ and $\left[x_{j}=V_{j}\right]$. Compare $V_{j}$ and $\varphi_{i, j}\left(V_{i}\right)$ :

- If $V_{j} \leq \varphi_{i, j}\left(V_{i}\right)$, then from the preliminary remark, all constraints satisfied by selector $\left[x_{i}=V_{i}\right]$ are satisfied by selector $\left[x_{j}=V_{j}\right]$ too. Hence selector $\left[x_{i}=V_{i}\right]$ can be suppressed - which contradicts the fact that $Z$ is maximal.

- Similarly if $\varphi_{i, j}\left(V_{i}\right) \leq V_{j}$, then all constraints satisfied by $\left[x_{j}=V_{j}\right]$ are satisfied by $\left[x_{i}=V_{i}\right]$, which contradicts the fact that $Z$ is maximal.

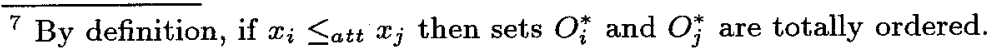


So $Z$ involves at most one among attributes $x_{i}$ and $x_{j}$.

- Suppose that $Z$ does not involve $x_{j}$. Then by construction, $Z$ belongs to G', and to $\mathrm{G}^{\prime \prime}$.

- Suppose that $Z$ involves $x_{j}$ and includes selector $\left[x_{j}=V_{j}\right]$. Let $V_{i}$ be such that

$$
V_{i}=\sup \left\{V_{k} / \varphi_{i, j}\left(V_{k}\right)=V_{j}\right\}
$$

Let $T$ be the term defined from $Z$ by replacing selector $\left[x_{j}=V_{j}\right]$ by $\left[x_{i}=V_{i}\right]$, which satisfies the same constraints by construction. We show that $T$ belongs to $G^{\prime}$.' In opposition, suppose that there exists $T^{\prime}$ in $\mathrm{G}^{\prime}$ such that $T^{\prime}>T$.

- If $T^{\prime}$ does not involve $x_{i}$, (as $T^{\prime}$ does not involve $x_{j}$ neither for $T^{\prime}$ belongs to $\mathrm{G}^{\prime}$ ), then from $T^{\prime}>T$ one has $T^{\prime}>Z$; this contradicts the fact that $Z$ is maximal.

- So $T^{\prime}$ must involve attribute $x_{i}$; assume that $T^{\prime}$ includes selector $\left[x_{i}=W_{i}\right]$ ; then $T^{\prime}>T$ implies $W_{i} \geq V_{i}$.

Consider now term $Z^{\prime}$, built by replacing in $T^{\prime}$ selector $\left[x_{i}=W_{i}\right]$ by $\left[x_{j}=\right.$ $\left.\varphi_{i, j}\left(W_{i}\right)\right]$. By definition of $\varphi_{i, j}, W_{i} \geq V_{i}$ implies $\varphi_{i, j}\left(W_{i}\right) \geq V_{j} ;$ hence $Z^{\prime} \geq Z$ ; however, $Z^{\prime}$ satisfies the same constraints as $Z$, and so belongs to G. But, $Z$ being maximal in $\mathrm{G}$, one has $Z=Z^{\prime}$, so $\varphi_{i, j}\left(W_{i}\right)=V_{j}$; by definition of $V_{i}$, this implies $V_{i} \geq W_{i}$, which contradicts the fact $T^{\prime} \neq T$.

Then there exists a term $T$ in $G^{\prime}$ such that $Z=T^{*}$. So, all terms in $\mathrm{G}$ are obtained from $\mathrm{G}^{\prime}$ by the procedure given in the proposition 2 .

\section{A.2 G" $\subset G$}

We show now that any term in G" belongs to G. By construction, if $T$ belongs to G' then it belongs to $\mathrm{G}$. It remains to show that all terms $T^{*}$ as defined in proposition 2 eventually belong to $\mathrm{G}$. Let $T$ be a term in $\mathrm{G}^{\prime}$ including selector $\left[x_{i}=V_{i}\right]$, with $V_{i}$ such that :

$$
V_{i}=\sup \left\{V_{k} / \varphi_{i, j}\left(V_{k}\right)=\varphi_{i, j}\left(V_{i}\right)\right\}
$$

Let $T^{*}$ be the term built from $T$ by replacing $\left[x_{i}=V_{i}\right]$ by $\left[x_{j}=\varphi_{i, j}\left(V_{i}\right)\right]$.

Suppose that there exists $Z$ in $\mathrm{G}$ such that $Z>T^{*}$.

- If $Z$ does not involve $x_{j}$, as $Z$ does not involve $x_{i}$ neither (for $Z>T^{*}$ and $T^{*}$ does not involve $x_{i}$ ), $Z$ belongs to $\mathrm{G}^{\prime}$. Hence $Z>T^{*}$ implies $Z>T$, which contradicts the fact that $T$ is maximal.

- If $Z$ involves $x_{j}$, then there exists in G' a term $S$ such that $Z=S^{*}$ (from A.1). It is straightforward to show that $Z=S^{*}>T^{*}$ implies $S \geq T$; now, as $T$ is maximal, $T=S$; so $Z=T^{*}$, and $T^{*}$ is maximal. 